\title{
Improving Value-at-Risk Estimation from the Normal EGARCH Model
}

\author{
Mahsa Gorji', Rasoul Sajjad ${ }^{2}$
}

ABSTRACT

Returns in financial assets display consistent excess kurtosis and skewness, implying the presence of large fluctuations not forecasted by Gaussian models. This paper applies a resampling method based on the bootstrap and a bias-correction step to improve Value-at-Risk (VaR) forecasting ability of the n-EGARCH (normal EGARCH) model and correct the VaR for both long and short positions. Our aim is to utilize the advantages of this model, but still use the bootstrap resampling method to accurate for the tendency of the model tomiscalculate the VaR. Empirical results indicate that the bias-correction method can improve the n-GARCH and n-EGARCH VaR forecasts so much that the acquired VaR predictions are different from the proposed probability. Additionally, allowing asymmetry in the conditional variance using the EGARCH model with normal distribution instead of GARCH improves the performance of the bias-correction method in forecasting the VaR for almost all considered indices. Moreover, the bias-corrected n-EGARCH model performs better than the simple t-EGARCH model. Thus, it seems that this model can take account of both the asymmetry in the conditional variance and leptokurtosis in returns distribution. However, we find that the superiority of the bias-corrected n-EGARCH model over the t-EGARCH model is not completely confirmed for short positions based on the censored likelihood scoring rule.

KEY WORDS: $\quad$ Bootstrap; EGARCH; GARCH; Value-at-Risk

JEL Classification: C58, G32, C15

${ }^{1}$ Faculty of Finance, University of Raja, Iran ${ }^{2}$ Faculty of Finance, University of Essex, Colchester, United Kingdom

\section{Introduction}

Since the Basle Committee $(1995 ; 1996)$ began allowing banks to implement internal VaR models for calculating capital requirements, various methods have been proposed to achieve this purpose. However, the theoretical and computational complexity of these methods has also been raised. Furthermore, various methods have been suggested for modeling conditional variance, and a large number of candidate distributions have been considered for modeling empirical

Correspondence concerning this article should be addressed to: Mahsa Gorji, T: 09126649690. E-mail: m.gorji@hotmail.com features of the returns (Alexander, 2001; Bao, Lee, \& Saltoğlu, 2007). Although more complex shapes of the tails have the potential preference of increased abilities to describe the VaR, they may lead to more uncertainty in the parameters and hence in the VaR estimate itself (Bams, Lehnert, \& Wolff, 2005).

The simple method that can consider two characteristics of financial asset returns, namely time-varying volatility and excess kurtosis, is the GARCH model by Engle (1982) and Bollerslev (1986). Researchers beginning with Black (1976) have demonstrated that stock returns are negatively correlated with changes in return volatility. Nevertheless, symmetric models such 
as the GARCH model have difficulties in correctly modeling the tails of the returns distribution (Giot \& Laurent, 2003) due to leverage effects. To overcome this shortcoming, various studies have proposed the inclusion different asymmetric terms in the conditional variance equation (Ding, Granger, \& Engle, 1993; Engle \& Ng, 1993; Glosten, Jagannathan, \& Runkle, 1993). Nelson (1991) also proposed the exponential GARCH model, which was re-expressed by Bollerslev and Mikkelsen (1996).

Hartz, Mittnik and Paolella (2006) have developed a resampling method based on the bootstrap and bias-correction for improving the VaR forecasting ability of the n-GARCH (normal GARCH) model. Their proposed method has improved the VaR forecasts of the n-GARCH model. Our main objective is to extend their study by allowing asymmetry in conditional variance. To this end, we apply the n-EGARCH in addition to the n-GARCH model to consider certain theoretical advantages of this model over the n-GARCH. However, Fama (1965) has demonstrated that return distributions of financial instruments are more leptokurtic than normal distributions and tend to be exhibit "fat tails". In addition, empirical studies of high-frequency financial time series demonstrate that the tail behavior of GARCH models remains too short even with standardized Student's $t$ innovations (Tsay, 2005). Therefore, we implement the bias-correction procedure based on the bootstrap method to remove the deficiencies of the n-GARCH and n-EGARCH models with respect to appropriate VaR forecasts. We try to conserve the simplicity of these methods, but still use the bootstrap resampling method to accurate for the tendency of these models to miscalculate the VaR. While Hartz et al. (2006), model the long VaR only, we try to extend their analysis by correcting the VaR for both long and short positions based on the aforementioned GARCH models (n-GARCH and n-EGARCH). Additionally, we evaluate models based on the censored likelihood (csl) scoring rule proposed by Diks, Panchenko, and Van Dijk (2011) in addition to the well-known Christoffersen's LR test. Empirical validation shows that considering asymmetry in conditional variance generally leads to improvements in accurately forecasting oneday-ahead VaR based on the bias-correction method for long and short positions, which is somewhat confirmed by the csl scoring rule.
The rest of the paper is organized as follows. Section 2 explains the methodology for estimating the distribution of the VaR point forecast and how it can be used to improve its accuracy. The empirical analysis and evaluation the performance of competing models in forecasting VaR are presented in Section 3. Finally, Section 4 concludes the paper.

\section{Forecasting VaR}

In general, for the set of equally spaced asset returns, $r_{t}, t=1, \ldots, T$, the class of $\operatorname{ARMA}(p, q)-\mathrm{EGARCH}$ $(r, s)$ models is given by

$r_{t}=a_{0}+\sum_{i=1}^{p} a_{i} r_{t-i}+\varepsilon_{t}+\sum_{j=1}^{q} b_{j} \varepsilon_{t-j}, \quad \varepsilon_{t}=z_{t} \sigma_{t}$,

$\ln \sigma_{t}^{2}=c_{0}+\sum_{i=1}^{r} c_{i} \ln \sigma_{t-i}^{2}+\sum_{j=1}^{s} d_{j}\left[\left|z_{t-j}\right|-E\left|z_{t-j}\right|\right]+\sum_{j=1}^{s} \theta_{j} z_{t-j}$

where $d_{j}$ represents the magnitude effect that indicates how much volatility increases autonomously of the direction of the shock. The $\theta_{j}$ is the sign effect. Both $z_{t}$ and $\left|z_{t}\right|-E\left|z_{t}\right|$ are zero-mean iid sequences with continuous distributions. $E\left|z_{t}\right|$ depends on the assumption made on the unconditional density of $z_{t}$. For the standard Gaussian random variable $t, E\left|z_{t}\right|=\sqrt{2 / \pi}$. The log of the conditional variance guarantees that forecasts of the conditional variance are non-negative (Nelson, 1991). Some additional properties of the EGARCH model can be found in Nelson (1991).

For a given return series and a chosen model from the n-ARMA-EGARCH class in (1) and (2), the usual conditional VaR forecast is acquired by estimating the unknown parameter vector $\theta=\left(a_{0}, \ldots, a_{p}, b_{1}, \ldots, b_{q}, c_{0}, \ldots, c_{r}, d_{1}, \ldots, d_{s}, \theta_{1}, \ldots, \theta_{s}\right) \quad$ via conditional maximum likelihood.

We also define the set of estimated standardized residuals $\left\{\hat{z}_{t}\right\}$, as $\hat{z}_{t}=\hat{\varepsilon}_{t} / \hat{\sigma}_{t}$, with

$$
\begin{aligned}
& \hat{\varepsilon}_{t}=r_{t}-\hat{a}_{0}-\left(\sum_{i=1}^{p} \hat{a}_{i} r_{t-i}\right)-\left(\sum_{j=1}^{q} \hat{b}_{j} \hat{\varepsilon}_{t-j}\right) \\
& \ln \hat{\sigma}_{t}^{2}=\hat{c}_{0}+\sum_{i=1}^{r} \hat{c}_{i} \ln \hat{\sigma}_{t-i}^{2}+\sum_{j=1}^{s} \hat{d}_{j}\left[\left|\hat{z}_{t-j}\right|-E\left|\hat{z}_{t-j}\right|\right]+\sum_{j=1}^{s} \hat{\theta}_{j} \hat{z}_{t-j}
\end{aligned}
$$

then the $h$-step-ahead (time horizon, $h \in \mathbb{Z}$ ) VaR forecast is obtained by

$\hat{v}_{\lambda}=\hat{v}_{\lambda}(h, T)=\Phi^{-1}\left(\lambda ; \hat{\mu}_{T+h}, \hat{\sigma}_{T+h}^{2}\right)$, 
where $\Phi^{-1}\left(\lambda ; \mu, \sigma^{2}\right)$, indicates the inverse cdf of the standard normal distribution with mean $\mu$ variance $\sigma^{2}$ and $\lambda$, a given probability level, and

$\hat{\mu}_{T+h}=\hat{a}_{0}+\sum_{i=1}^{p} \hat{a}_{i} r_{T+h-i}+\sum_{j=1}^{q} \hat{b}_{j} \hat{\varepsilon}_{T+h-j}$

$\ln \hat{\sigma}_{T+h}^{2}=\hat{c}_{0}+\sum_{i=1}^{r} \hat{c}_{i} l n \hat{\sigma}_{T+h-i}^{2}+\sum_{j=1}^{s} \hat{d}_{j}\left[\left|\hat{z}_{T+h-j}\right|-E\left|z_{t-j}\right|\right]+\sum_{j=1}^{s} \hat{\theta}_{j} \hat{z}_{T+h-j}$

The VaR for a short position is similarly computed where the same definition is used for the right tail of the distribution function, i.e., $1-\lambda$ substitutes for $\lambda$.

\subsection{VaR forecast distribution}

For implementing the bias-correction method we need to estimate VaR forecast distribution in addition to a VaR point estimator $\left(\hat{v}_{\lambda}\right)$. Therefore, as Hartz et al. (2006) proposed, we apply the bootstrap method to this end. This method coincides with that described in Pascual, Romo and Ruiz (2006), Reeves (2005) and Trucíos and Hotta (2016). It is connected to the filtered historical simulation method proposed by BaroneAdesi, Giannopoulos and Vosper $(1999 ; 2002)$ and the bootstrap methodology described by Dowd (2005).

Despite that the sampling distribution of the $\mathrm{VaR}$ point forecast is unknown and intangible, the bootstrap method provides the possibility for its approximation. Because, assuming the true data generating process is constant over time, the bias caused by the use of the inappropriate but simple n-GARCH model will display certain regularities and thus can be corrected based on a set of past VaR bootstrap distributions (Hartz et al., 2006). This is the assumption used in the bias-correction method described in Section 2.2.

For implementing $B$ bootstrap iterations, the $b$ th replication, $b=1, \ldots, B$, we should consider the following steps as Hartz et al. (2006) suggested:

Step 0: For a chosen set of values $p, q, r, s$ (for which $p=r=s=1$ and $q=0$ is most common), obtain QML parameter vector estimate $\hat{\theta}$, estimated standardized residuals $\left\{\hat{z}_{t}\right\}$, then forecast $\mathrm{VaR}$ for a certain $h$ (we consider $h=1$ ) by (5).

Step 1: Simulate the $\left(b^{\prime}\right)$ th of $B, \mathrm{n}-\operatorname{ARMA}(p, q)$ $\operatorname{EGARCH}(r, s)$ time series, $\left\{r_{t}^{\left(b^{\prime}\right)}\right\}$, of length $T$, using the estimated parameter vector $\hat{\theta}$ and bootstrapped standardized residuals sequence $\left\{\hat{z}_{t}^{\left(b^{\prime}\right)}\right\}$. To eliminate the effect of initial values, we simulate $T+\ell$ series and then discard first $\ell$ observations $(\ell=T)$.
Step 2: Obtain the QML parameter vector estimate $\hat{\theta}^{\left(b^{\prime}\right)}$ based on n-ARMA $(p, q)$-EGARCH $(r, s)$ using the simulated time series $\left\{r_{t}^{\left(b^{\prime}\right)}\right\}$.

Step 3: Estimate a resampled VaR estimate, $\hat{v}_{\lambda}^{(b)}(h, T)$, using the original series $\left\{r_{t}\right\}$, and the bootstrap parameter vector estimate $\hat{\theta}^{\left(b^{\prime}\right)}$, via

$\hat{\varepsilon}_{t}^{(b)}=r_{t}-\hat{a}_{0}^{(b)}-\left(\sum_{i=1}^{p} \hat{a}_{i}^{(b)} r_{t-i}\right)-\left(\sum_{j=1}^{q} \hat{b}_{j}^{(b)} \hat{\varepsilon}_{t-j}^{(b)}\right), \quad t=1, \ldots, T$,

$\ln \hat{\sigma}_{t}^{2(b)}=\hat{c}_{0}^{(b)}+\sum_{i=1}^{r} \hat{c}_{i}^{(b)} \ln \hat{\sigma}_{t-i}^{2(b)}+\sum_{j=1}^{s} \hat{d}_{j}^{(b)}\left[\left|\hat{z}_{t-j}^{(b)}\right|-E\left|z_{t-j}\right|\right]+$

$+\sum_{j=1}^{s} \hat{\theta}_{j}^{(b)} \hat{z}_{t-j}^{(b)}$

and to calculate bootstrapped VaR forecast

$\hat{v}_{\lambda}^{(b)}(h, T)=\Phi^{-1}\left(\lambda, \hat{\mu}_{T+h}^{(b)}, \hat{\sigma}_{T+h}^{2(b)}\right)$,

where

$\hat{\mu}_{T+h}^{(b)}=\hat{a}_{0}^{(b)}+\sum_{i=1}^{p} \hat{a}_{i}^{(b)} r_{T+h-i}+\sum_{j=1}^{q} \hat{b}_{j}^{(b)} \hat{\varepsilon}_{T+h-j}^{(b)}$,

$\ln \hat{\sigma}_{T+h}^{2(b)}=\hat{c}_{0}^{(b)}+\sum_{i=1}^{r} \hat{c}_{i}^{(b)} \ln \hat{\sigma}_{T+h-i}^{2(b)}+\sum_{j=1}^{s} \hat{d}_{j}^{(b)}\left[\left|\hat{z}_{T+h-j}^{(b)}\right|-E\left|z_{t-j}\right|\right]+$

$+\sum_{j=1}^{s} \hat{\theta}_{j}^{(b)} \hat{z}_{T+h-j}^{(b)}$,

are calculated using conditional expectations for unobserved values.

The primary VaR prediction, $\hat{v}_{\lambda}^{(0)}$, and the $B$ bootstrapped VaR predictions, $\hat{v}_{\lambda}^{(b)}, b=1, \ldots, B$, can be used to construct an empirical distribution function of VaR estimation. This function is given by

$\hat{F}_{\hat{k}}(a ; \lambda, h, T)=\frac{1}{B+1} \sum_{b=0}^{B} \psi_{(-\infty, a)}\left(\hat{v}_{\lambda}^{(b)}(h, T)\right)$,

where $\psi($.$) is the indicator function. This function$ could be used to construct a bootstrap confidence interval for the VaR; for instance Christoffersen and Goncalves (2005) propose to use the bootstrap for constructing confidence intervals of a conditional VaR estimator. Nieto and Ruiz (2010) suggest a new bootstrap procedure to obtain prediction intervals of future VaR and Expected Shortfall (ES), as well. We use the bootstrap method as described in the previous section to obtain a more accurate VaR based on n-GARCH models (n-GARCH and n-EGARCH) as much as possible. 


\subsection{Bias-correcting VaR forecasts}

The corrective method uses the VaR distributions (approximated based on the illustrated bootstrapped method) and an objective function, which is described by definition of the VaR. The VaR is defined to be the worst possible loss from an investment over a target horizon and for a given probability level (Crouhy, Galai, \& Mark, 2001). Therefore, the evident criterion to construct this function is the observed frequency of exceptions, or past realized returns that are less (higher) than or equal to the predicted VaR for long (short) positions.

For a given probability level $\lambda$, the observed frequency of exceptions, denoted $\hat{f}$, for a set of successive VaR predictions for long positions obtained from the usual n-GARCH models between times, say, $\tau_{1}$ and $\tau_{2}$, and the equivalent realized returns is given by

$\hat{f}=\frac{1}{\tau_{2}-\tau_{1}+1} \sum_{t=\tau_{1}}^{\tau_{2}} \psi_{(-\infty, \hat{v}(t)]}\left(r_{t+h}\right), \quad \hat{v}(t)=\hat{v}_{\lambda}(h, t)$

The observed frequency of exceptions for a short position is similarly computed where the same definition is used for the right tail of the distribution function, i.e., $\psi_{[\hat{v}(t),+\infty)}$ substitutes for $\psi_{(-\infty, \hat{v}(t)]}$.

The observed frequency of exceptions is less (higher) than the real risk level $\lambda$, if the VaR forecasts calculated by the n-GARCH models tend to overestimate (underestimate) the risk. Therefore, the logic behind the bias-correction method is to find the quantile of past VaR distributions which causes observed frequency of exceptions conforms to (as close as possible) a given risk level.

Let $\left\{\hat{v}_{\lambda}^{[b]}(h, t)\right\}_{b=0}^{B}$ be the sorted VaR predictions, with length $(B+1)$ produced by the resampling algorithm with the original n-GARCH models forecast, $\hat{v}_{\lambda}^{(0)}(h, t)$, added, i.e., $\hat{v}_{\lambda}^{[b]}(h, t) \leq \hat{v}_{\lambda}^{[b+1]}(h, t)$ for $b=0, \ldots, B-1$. Calculating the correct quantile of the VaR distribution $\hat{F}_{\hat{k}}$ is equivalent to find the largest index $b$ for the long VaR (the smallest for the short VaR), denoted $b^{*}$, such that for the conforming series $\left\{\hat{v}_{\lambda}^{\left[b^{*}\right]}(h, t)\right\}_{t=\tau_{1}}^{\tau_{2}}$, the observed frequency of exceptions is less than or equal to the given risk level. Therefore, we need a certain number of past VaR distribution predictions that precede the actual VaR prediction of interest. As Hartz et al. (2006) have described, we consider a moving window procedure for finding the proper quantile of the VaR distribution produced by the resampling al- gorithm. Common to all models is the construction of a moving window in which the model is recalculated for each window period, removing the first observations and adding new ones as the window advances. $L$ is the fixed number of preceding VaR forecast distributions that we consider for finding the proper quantile for the $h$-step-ahead prediction for the downfall risk made at time $T$. Accordingly, the optimal quantile is described as

$b^{*}=\max \{b: b \in\{0,1, \ldots, B\}\}$ s.t. $\frac{1}{L} \sum_{t=T-L-h+1}^{T-h} \psi_{\left(-\infty, i\left[i^{[l]}(h, t)\right)\right.}\left(r_{t+h}\right) \leq \lambda$.

where $b^{*}$ denotes the greatest quantile of the last $L$ feasible VaR distributions for which the conforming series of VaR predictions, $\left.\left\{\hat{v}_{\lambda}^{b^{*}}\right](h, t)\right\}$, leads to an observed frequency of VaR exceptions that is equal to (or just smaller) than the given risk level. The $b^{*}$ for a short position is similarly computed where it determines the smallest quantile of the VaR distributions and the $\psi_{\left(\hat{i}^{[b]}(h, t), \infty\right)}$ substitutes for $\left.\psi_{(-\infty, \hat{i}[\hat{b}]}(h, t)\right)$.

As the data generating process is not constant over time, calculating an optimal $L$ would be reliable only for particular segment of a particular data set (Hartz et al., 2006). Thus, following the suggestion of Hartz et al. (2006) concerning the choice of $L$, we assume two sizes of the moving window $(L)$ to examine whether this criterion could affect the results of our study.

We consider two values, $L=250$ and 500, (one and two years of trading data, respectively) to perform the bias-correction method.

\section{Empirical analysis}

We consider the daily percentage log-return series, defined by $r_{t}=\left(\ln p_{t}-\ln p_{t-1}\right) \times 100$, where $p_{t}$ is the closing price index on day $t$. The data set analyzed in this paper comprises daily observations on three major stock market indices' returns; namely, the FTSE Composite, NIKKEI 225 and CAC (hereafter the FTSE, NIKKEI and CAC). The starting date for the series are August 27, 1999 for the FTSE; April 09, 1999 for the NIKKEI; and November 05, 1999 for the CAC.

The usual descriptive statistics of the data are given in Table 1. The moments of the stock index returns are shown along with the results of an aggregate autocorrelation (Ljung-Box) test for returns and their squares. As seen, for the FTSE and NIKKEI the skewness is significant and negative, indicating a possible leverage 
Table 1. Moments of the FTSE, NIKKEI and CAC returns along with aggregate autocorrelation test results.

\begin{tabular}{|c|c|c|c|}
\hline & FTSE & NIKKEI & CAC \\
\hline Mean & 0.0008 & -0.004 & -0.007 \\
\hline Minimum & -9.26 & -12.11 & -9.47 \\
\hline Maximum & 9.38 & 13.23 & 10.59 \\
\hline Std. Dev. & 1.276 & 1.565 & 1.550 \\
\hline Skewness & -0.126 & -0.419 & 0.028 \\
\hline Kurtosis & 8.715 & 9.361 & 7.540 \\
\hline \multicolumn{4}{|c|}{ Ljung-Box test for returns } \\
\hline \multirow[t]{2}{*}{ Q-stat(12) } & 78.81 & 13.85 & 56.95 \\
\hline & $\{0\}$ & $\{0.31\}$ & $\{0\}$ \\
\hline \multirow[t]{2}{*}{ Q-stat(24) } & 102.2 & 44.34 & 71.28 \\
\hline & $\{0\}$ & $\{0.007\}$ & $\{0\}$ \\
\hline \multirow[t]{2}{*}{ Q-stat(36) } & 153.52 & 61.00 & 120.97 \\
\hline & $\{0\}$ & $\{0.006\}$ & $\{0\}$ \\
\hline \multicolumn{4}{|c|}{ Ljung-Box test for squared returns } \\
\hline \multirow[t]{2}{*}{ Q-stat(12) } & 2797 & 3051 & 1988 \\
\hline & $\{0\}$ & $\{0\}$ & $\{0\}$ \\
\hline \multirow[t]{2}{*}{ Q-stat(24) } & 4340 & 3860 & 3138 \\
\hline & $\{0\}$ & $\{0\}$ & $\{0\}$ \\
\hline \multirow[t]{2}{*}{ Q-stat(36) } & 5351 & 4114 & 3907 \\
\hline & $\{0\}$ & $\{0\}$ & $\{0\}$ \\
\hline
\end{tabular}

Note:

p-values in curly braces.

Q-stat (q) denotes a modified Ljung-Box type statistic.

effect in data, but for the CAC the skewness is positive, and the kurtosis is significantly higher than that of a Gaussian distribution (excess kurtosis), indicating fat-tailed returns for all series. This suggests the need for a fat-tailed distribution, for example Student's $t$, to describe the returns' conditional distribution. Furthermore, imposing an asymmetric parameter in the conditional variance equation enables us to capture possible leverage effects in the data. In addition, the large Q-statistics up to 12,24 and 36 orders strongly reject the null hypothesis of no serial correlation in both returns and squared returns for the FTSE and CAC but only in returns for the NIKKEI index.

The estimation period is set to $T=1000$, which corresponds to about four years of daily returns data for estimating the parameters of models. $B=1000$ bootstrap replications are used for calculating the VaR forecast distributions.

For each series, we use $p=2000$ out-of-sample values, and the last forecast is made for July 16, 2013 for 
each series. The models are used to estimate one-dayahead VaR $(h=1)$ of both long and short trading positions (left and right tails of returns distribution) with different probabilities (at different tail quantiles): $0.5 \%$, $1 \%, 5 \%, 95 \%$, $99 \%$ and $99.5 \%$.

For implementing the bias-correction method, we use the two values $L=250$ and 500. So, an additional number of $L=500 \mathrm{VaR}$ forecast distributions resulting in a total of $P+L=2500 \mathrm{VaR}$ forecast distributions are considered.

In addition to the $\mathrm{n}-\mathrm{AR}(1)-\mathrm{GARCH}(1,1)$ and $\mathrm{n}$-AR(1)-EGARCH $(1,1)$ models, we consider the $\operatorname{AR}(1)-E G A R C H(1,1)$ with the Generalized Error Distribution (GED) and t-AR(1)-EGARCH(1,1) (with Student's $t$ innovations) to assess the performance of the bias-correction method. ${ }^{1}$ The original form of this model is used for the comparison due to its ability to consider the asymmetry in the conditional variance and the excess kurtosis relative to the normal distribution. In this model, the conditional variance obtained is similar to the n-EGARCH model (i.e., Equation (2)), while the $E\left|z_{t}\right|$ for a Student's t distribution with $v>2$ degrees of freedom is given by

$E\left(\left|z_{t}\right|\right)=\frac{2 \sqrt{v-2} \Gamma((v+1) / 2)}{(v-1) \Gamma(v / 2) \sqrt{\pi}}$,

where $\Gamma(x)$ is the usual gamma function.

The corresponding VaR prediction for the t-AR(1)$\operatorname{EGARCH}(1,1)$ model is given by

$\hat{v}_{\lambda}=\hat{v}_{\lambda}(h, T)=\hat{\mu}_{T+h}+\hat{\sigma}_{T+h} F_{t}^{-1}(\lambda ; \hat{v})$,

where $\mathrm{F}_{\mathrm{t}}^{-1}(\lambda ; v)$ is the inverse of the cumulative distribution function of the $t$-distribution with $v$ degrees of freedom and a standardized variance of one.

In the remainder of the paper, we first examine the $\mathrm{VaR}$ forecast distributions generated by the bootstrap algorithm. Then, we compare the VaR estimations of the competing models.

\subsection{VaR forecast distribution}

Figures 1-3 present the first VaR forecast distributions included in our analysis for the FTSE, NIKKEI and CAC for both long and short trading positions. The upper part of the figures refers to the VaR forecast distribution for the n-GARCH model, while the lower part of the figures refers to the VaR forecast distribution for the n-EGARCH model. Additionally, the vertical lines exhibit the usual n-GARCH and nEGARCH VaR predictions, $\hat{v}^{0}$.

As seen from figures 1, 2 and 3, the risk in VaR forecasts for both long and short trading positions increases for smaller probability levels. The usual VaR predictions do not exactly coincide with the mode of the VaR forecast distributions. For instance, for the first VaR forecasts made for all series, the usual n-EGARCH $\mathrm{VaR}$ forecasts are smaller than the mode of the VaR forecast distributions for both long and short trading positions. On the other hand, the first VaR forecasts for all series from the usual n-GARCH model for short trading position are greater than the mode of the corresponding VaR forecast distributions.

\subsection{Performance of Models in Forecasting VaR}

For assessing the accuracy of competing methods, we define the Boolean sequence as

$I_{t+1}=\psi_{\left(-\infty, \hat{v}_{\lambda}(1, t)\right)}\left(r_{t+1}\right)$,

where $\hat{v}_{\lambda}(1, t)$ is one-step-ahead VaR predictions for $\lambda$, a given probability level, and $r_{t+1}$ is the observed return. Christoffersen (1998) showed that evaluating interval forecasts can be reduced to examining whether the Boolean sequence, $\left\{I_{t}\right\}_{t=1}^{T}$, satisfies the unconditional coverage (UC) and independence (IND) properties.

Let $T_{1}=\sum_{t=1}^{T} I_{t+1}$, the number of violations, and the failure rate be expressed as $\hat{f}=T^{-1} \sum_{t=1}^{T} I_{t+1}=T_{1} / T$. For testing the UC, the proper likelihood ratio statistic, also called the Kupiec (1995) LR unconditional test, (under the null hypothesis that, $\hat{f}=\lambda$ ) is

$$
\begin{aligned}
& L R_{u c}=2 \ln \left[(1-\hat{f})^{T-T_{1}}(\hat{f})^{T_{1}}\right]-2 \ln \left[(1-\lambda)^{T-T_{1}}(\lambda)^{T_{1}}\right] \sim \chi_{1}^{2}, \\
& P_{u n}=1-F_{\chi_{1}^{2}}\left(L R_{u n}\right),
\end{aligned}
$$

For $P_{u n}$ below the desired significance level, the null hypothesis is rejected.

The $L R_{c c}$ test adapted from Christoffersen (1998) is used to test the conditional coverage.

We compare models based on the $p$-values, and models with higher $p$-values are preferred. 


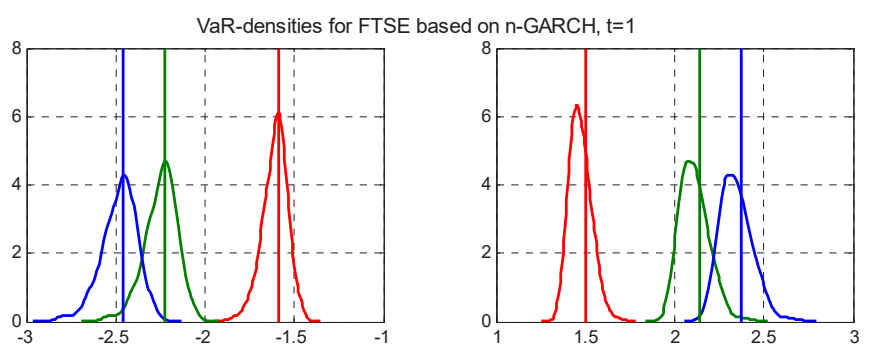

VaR-densities for FTSE based on n-EGARCH, t=1

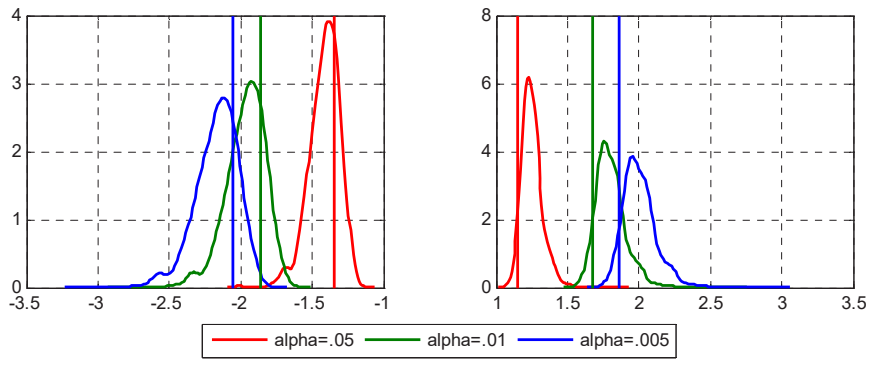

Figure 1. Vertical lines refer to the usual $n-G A R C H$ and $n-E G A R C H$ VaR predictions, $\hat{v}^{0}$. VaR forecast distributions for the first forecast - FTSE.

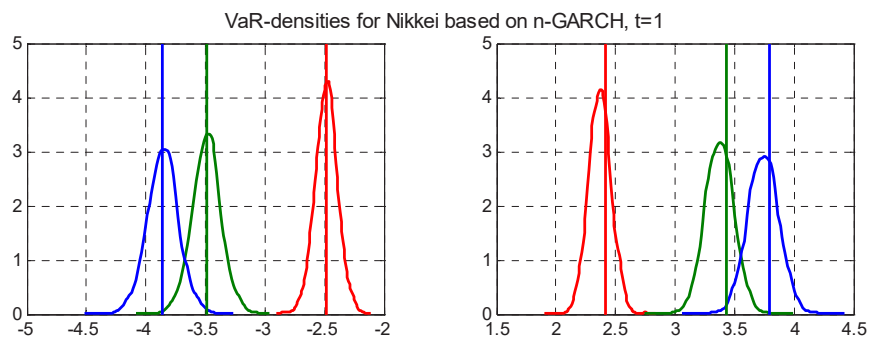

VaR-densities for Nikkei based on $n-E G A R C H, t=1$
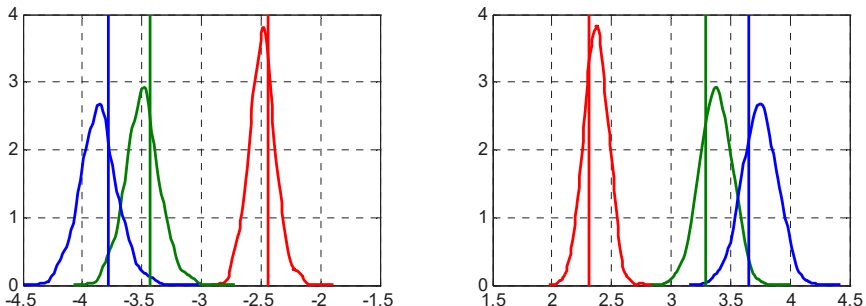

alpha $=.05-$ alpha $=.01-$ alpha $=.005$

Figure 2. Vertical lines refer to the usual $n-G A R C H$ and $n-E G A R C H$ VaR predictions, $\hat{v}^{0}$. VaR forecast distributions for the first forecast - NIKKEI. 

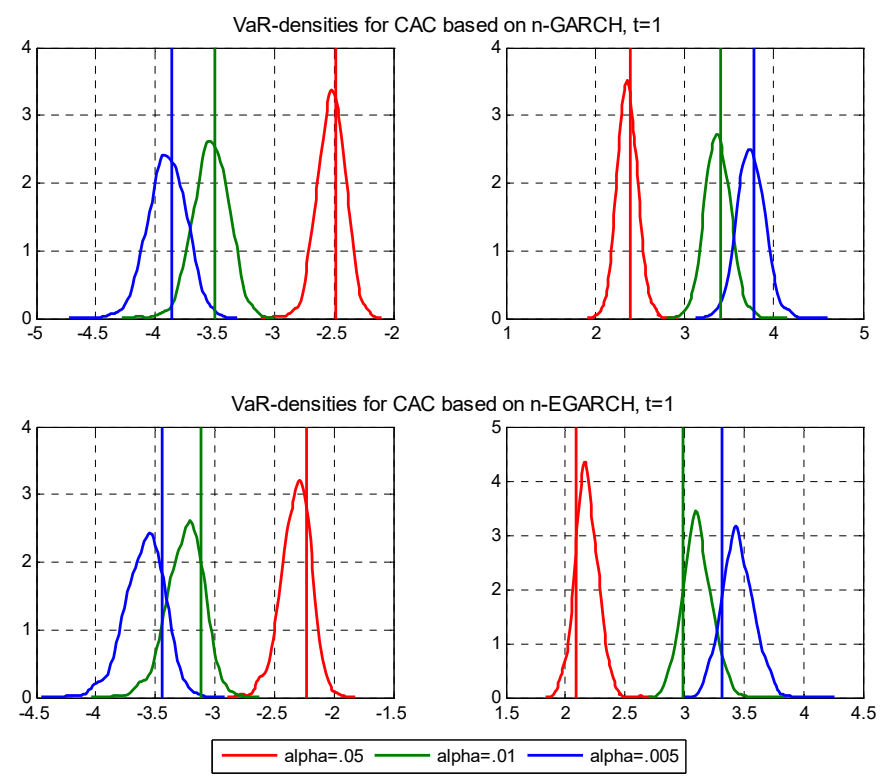

Figure 3. Vertical lines refer to the usual $n-G A R C H$ and $n-E G A R C H$ VaR predictions, $\hat{v}^{0}$. VaR forecast distributions for the first forecast - CAC.

In addition to unconditional and conditional tests we consider a csl scoring rule suggested by Diks et al. (2011) to assess the performance of the bias-correction method. They have shown that this scoring rule is handy as the main interest lies in comparing the accuracy of density forecasts for a specific region, such as the left tail in financial risk management. While they have considered long VaR only, we also evaluate our models for both long and short positions. The censored likelihood (csl) score function is given by

$\mathrm{S}^{\mathrm{csl}}\left(\hat{\mathrm{a}}_{\mathrm{t}} ; \mathrm{r}_{\mathrm{t}+1}\right)=\mathrm{w}_{\mathrm{t}}\left(\mathrm{r}_{\mathrm{t}+1}\right) \log \hat{\mathrm{a}}_{\mathrm{t}}\left(\mathrm{r}_{\mathrm{t}+1}\right)+$

$+\left(1-\mathrm{w}_{\mathrm{t}}\left(\mathrm{r}_{\mathrm{t}+1}\right)\right) \log \left(1-\int \mathrm{w}_{\mathrm{t}}(\mathrm{s}) \hat{\mathrm{a}}_{\mathrm{t}}(\mathrm{s}) \mathrm{ds}\right)$,

$\mathrm{d}_{\mathrm{t}+1}^{\mathrm{csl}}=\mathrm{S}^{\mathrm{csl}}\left(\hat{\mathrm{a}}_{\mathrm{t}} ; \mathrm{r}_{\mathrm{t}+1}\right)-\mathrm{S}^{\mathrm{csl}}\left(\hat{\mathrm{b}}_{\mathrm{t}} ; \mathrm{r}_{\mathrm{t}+1}\right)$

where $\hat{a}_{t}$ and $\hat{b}_{t}$ are two competing density forecasts and $\mathrm{w}_{\mathrm{t}}(\mathrm{r})$ is a threshold weight function $\mathrm{w}_{\mathrm{t}}(\mathrm{r})=\mathrm{I}\left(\mathrm{r} \leq \hat{\mathrm{V}}_{\mathrm{t}}^{\alpha}\right)$. Additionally, corresponding realizations of the variable $r_{t+1}$ are accessible for $t=n, n+1, \ldots, T-1$. Then, $\hat{a}_{t}$ and $\hat{b}_{t}$ are comparable based on their average scores difference $d$, by testing if their difference is statistically significant ${ }^{2}$.

In the following, we first concentrate on the measures $L R_{c c}, L R_{\text {ind }}$ and $L R_{u c}$. The results for the seven competing models are given in Tables 2-4 for the FTSE, NIKKEI and CAC.

We first examine the capability of the bias-correction procedure to improve the VaR forecasts of the usual n-GARCH and n-EGARCH models. Comparing the forecasts from the usual models with the biascorrected forecasts, we find that the $P_{u c}$-values for at least five out of the 6 specified probabilities for $L=500$ are superior (larger) to those for the usual n-GARCH model, while for the n-EGARCH model the $p$-values for at least four out of the 6 cases for both window lengths are superior to those for the usual n-EGARCH model.

We found no $P_{u c}$-values below the 5\% significance level for the bias-corrected VaR predictions of the nEGARCH model with $L=500$ for all series except the NIKKEI at $99 \%$, while for the usual VaR forecasts, we 
Table 2. Unconditional coverage, independence, conditional coverage-FTSE.

\begin{tabular}{|c|c|c|c|c|c|c|c|c|c|}
\hline \multirow{2}{*}{$\begin{array}{l}\text { VaR } \\
\text { models }\end{array}$} & \multirow{2}{*}{$\hat{f}$} & \multicolumn{3}{|c|}{$p$-values } & \multirow{2}{*}{$\hat{f}$} & \multicolumn{3}{|c|}{$p$-values } & \multirow[b]{2}{*}{$* / * * / * * *$} \\
\hline & & $P_{c c}$ & $P_{i n d}$ & $P_{u n}$ & & $P_{c c}$ & $P_{\text {ind }}$ & $P_{u n}$ & \\
\hline & \multicolumn{4}{|c|}{ VaR 5\% } & \multicolumn{4}{|c|}{ VaR 95\% } & \\
\hline$\hat{f}_{N}$ & 0.0645 & 0.0133 & 0.4802 & 0.0043 & 0.0420 & 0.0600 & 0.0952 & 0.0918 & $5 / 3 / 3$ \\
\hline$\hat{f}_{N-500}^{*}$ & 0.0545 & 0.4343 & 0.3598 & 0.3624 & 0.0495 & 0.0794 & 0.0245 & 0.9182 & $2 / 1 / 0$ \\
\hline$\hat{f}_{E G A R C H}$ & 0.0605 & 0.1052 & 0.7070 & 0.0367 & 0.0435 & 0.0803 & 0.0742 & 0.1730 & $4 / 4 / 2$ \\
\hline$\hat{f}_{E G A R C H-500}^{*}$ & 0.0505 & 0.6627 & 0.3674 & 0.9184 & 0.0520 & 0.1870 & 0.0742 & 0.6834 & 0/0/0 \\
\hline \multirow[t]{2}{*}{$\hat{f}_{t-E G A R C H}$} & 0.0650 & 0.0120 & 0.6866 & 0.0032 & 0.0450 & 0.0951 & 0.0572 & 0.2970 & $5 / 5 / 4$ \\
\hline & \multicolumn{4}{|c|}{ VaR 1\% } & \multicolumn{4}{|c|}{ VaR 99\% } & \\
\hline$\hat{f}_{N}$ & 0.0210 & $<0.0001$ & 0.2852 & $<0.0001$ & 0.0070 & 0.3258 & 0.6456 & 0.1541 & \\
\hline$\hat{f}_{N-500}^{*}$ & 0.0145 & 0.0321 & 0.0699 & 0.0581 & 0.0130 & 0.3054 & 0.3990 & 0.1974 & \\
\hline$\hat{f}_{E G A R C H}$ & 0.0205 & 0.0001 & 0.7898 & $<0.0001$ & 0.0075 & 0.4440 & 0.6229 & 0.2397 & \\
\hline$\hat{f}_{E G A R C H-500}^{*}$ & 0.0135 & 0.2235 & 0.3813 & 0.1353 & 0.0120 & 0.5049 & 0.4357 & 0.3835 & \\
\hline$\hat{f}_{t-E G A R C H}$ & 0.0210 & $<0.0001$ & 0.8094 & $<0.0001$ & 0.0050 & 0.0429 & 0.7395 & 0.0129 & \\
\hline
\end{tabular}

\begin{tabular}{|c|c|c|c|c|c|c|c|c|}
\hline \multirow[b]{2}{*}{$\hat{f}_{N}$} & \multicolumn{4}{|c|}{ VaR 0.5\% } & \multicolumn{4}{|c|}{ VaR 99.5\% } \\
\hline & 0.0155 & $<0.0001$ & 0.4905 & $<0.0001$ & 0.0025 & 0.2111 & 0.8623 & 0.0792 \\
\hline$\hat{f}_{N-500}^{*}$ & 0.0095 & 0.0331 & 0.5356 & 0.0112 & 0.0070 & 0.4403 & 0.6456 & 0.2319 \\
\hline$\hat{f}_{E G A R C H}$ & 0.0115 & 0.0015 & 0.4548 & 0.0004 & 0.0020 & 0.0950 & 0.8874 & 0.0304 \\
\hline$\hat{f}_{E G A R C H-500}^{*}$ & 0.0060 & 0.7654 & 0.6920 & 0.5388 & 0.0045 & 0.9074 & 0.7636 & 0.7471 \\
\hline$\hat{f}_{t-E G A R C H}$ & 0.0120 & 0.0006 & 0.4357 & 0.0001 & 0.0010 & 0.0082 & 0.9382 & 0.0020 \\
\hline
\end{tabular}

Note: $\hat{f}_{N}$ : observed downfall probability for $n-A R(1)-G A R C H(1,1)$ model; $\hat{f}_{E G A R C H}$ : observed downfall probability for $n$-AR(1)$\operatorname{EGARCH}(1,1)$ model; $\hat{f}_{t-E G A R C H}$ : observed downfall probability for $t-A R(1)-E G A R C H(1,1)$ model; $\hat{f}_{N-500}^{*}\left(\hat{f}_{E G A R C H-500}^{*}\right)$ : observed downfall probability for calibrated $n-A R(1)-G A R C H(1,1)(n-A R(1)-E G A R C H(1,1))$ model with $L=500 ; P_{u n}\left(P_{\text {ind }}, P_{c c}\right)$ : probability of observing a sample with higher unconditional coverage test statistic (independence test statistic, conditional coverage test statistic). The results for both long and short positions are reported in the left and right panel of the table, respectively. ******. Number of p-values for unconditional coverage are smaller than 0.10/0.05/0.01.

have at least $2 p$-values below the $1 \%$ level and one $p$ value below the $0.01 \%$.

The results show that the usual VaR models based on normal innovations have difficulties with modeling large returns. In particular, the usual normal GARCH models consistently underestimate the return (risk) for all series for long positions. In other words, failure numbers are much greater than expected at a given quantile in the case of normal innovations.

Regarding independence of the VaR exceptions over time, for both the usual n-GARCH and n-EGARCH $\mathrm{VaR}$ forecast violations or those from the bias-correc- 
Table 3. Unconditional coverage, independence, conditional coverage-NIKKEI.

\begin{tabular}{|c|c|c|c|c|c|c|c|c|c|}
\hline \multirow{2}{*}{$\begin{array}{l}\text { VaR } \\
\text { models }\end{array}$} & \multirow{2}{*}{$\hat{f}$} & \multicolumn{3}{|c|}{$p$-values } & \multirow{2}{*}{$\hat{f}$} & \multicolumn{3}{|c|}{$p$-values } & \multirow[b]{2}{*}{$* / * * * * *$} \\
\hline & & $P_{c c}$ & $P_{\text {ind }}$ & $P_{u n}$ & & $P_{c c}$ & $P_{\text {ind }}$ & $P_{u n}$ & \\
\hline & \multicolumn{4}{|c|}{ VaR 5\% } & \multicolumn{4}{|c|}{ VaR 95\% } & \\
\hline$\hat{f}_{N}$ & 0.0605 & 0.1024 & 0.6599 & 0.0367 & 0.0430 & 0.2589 & 0.4619 & 0.1415 & $5 / 5 / 3$ \\
\hline$\hat{f}_{N-500}^{*}$ & 0.0540 & 0.4759 & 0.3629 & 0.4175 & 0.0570 & 0.2923 & 0.4874 & 0.1596 & 0/0/0 \\
\hline$\hat{f}_{E G A R C H}$ & 0.0595 & 0.1415 & 0.5720 & 0.0581 & 0.0550 & 0.2789 & 0.2157 & 0.3123 & $4 / 2 / 2$ \\
\hline$\hat{f}_{E G A R C H-500}^{*}$ & 0.0555 & 0.3158 & 0.3001 & 0.2671 & 0.0595 & 0.0537 & 0.1329 & 0.0581 & $2 / 1 / 0$ \\
\hline$\hat{f}_{t-E G A R C H}$ & 0.0620 & 0.0552 & 0.7064 & 0.0174 & 0.0555 & 0.1617 & 0.1204 & 0.2671 & $4 / 3 / 1$ \\
\hline
\end{tabular}

\begin{tabular}{|c|c|c|c|c|c|c|c|c|}
\hline \multirow[b]{2}{*}{$\hat{f}_{N}$} & \multicolumn{4}{|c|}{ VaR 1\% } & \multicolumn{4}{|c|}{ VaR 99\% } \\
\hline & 0.0160 & 0.0123 & 0.1036 & 0.0131 & 0.0045 & 0.0205 & 0.7636 & 0.0056 \\
\hline$\hat{f}_{N-500}^{*}$ & 0.0125 & 0.3355 & 0.3138 & 0.2794 & 0.0130 & 0.3054 & 0.3990 & 0.1974 \\
\hline$\hat{f}_{E G A R C H}$ & 0.0220 & $<0.0001$ & 0.3342 & $<0.0001$ & 0.0070 & 0.3258 & 0.6456 & 0.1541 \\
\hline$\hat{f}_{E G A R C H-500}^{*}$ & 0.0130 & 0.2771 & 0.3413 & 0.1974 & 0.0150 & 0.0242 & 0.0801 & 0.0364 \\
\hline$\hat{f}_{t-E G A R C H}$ & 0.0180 & 0.0049 & 0.6493 & 0.0012 & 0.0055 & 0.0812 & 0.7156 & 0.0270 \\
\hline
\end{tabular}

\begin{tabular}{|c|c|c|c|c|c|c|c|c|}
\hline \multirow[b]{2}{*}{$\hat{f}_{N}$} & \multicolumn{4}{|c|}{ VaR 0.5\% } & \multicolumn{4}{|c|}{ VaR 99.5\% } \\
\hline & 0.0100 & 0.0087 & 0.1918 & 0.0053 & 0.0010 & 0.0082 & 0.9382 & 0.0020 \\
\hline$\hat{f}_{N-500}^{*}$ & 0.0060 & 0.1392 & 0.0590 & 0.5388 & 0.0065 & 0.6037 & 0.6686 & 0.3634 \\
\hline$\hat{f}_{E G A R C H}$ & 0.0110 & 0.0023 & 0.2373 & 0.0010 & 0.0025 & 0.2111 & 0.8623 & 0.0792 \\
\hline$\hat{f}_{E G A R C H-500}^{*}$ & 0.0050 & 0.1172 & 0.0384 & 1.0000 & 0.0070 & 0.4403 & 0.6456 & 0.2319 \\
\hline$\hat{f}_{t-E G A R C H}$ & 0.0075 & 0.0866 & 0.0993 & 0.1401 & 0.0025 & 0.2111 & 0.8623 & 0.0792 \\
\hline
\end{tabular}

Note: See the legend of Table 2 for explanations.

tion method, independence cannot be rejected; in other words, the occurrence of violations for VaR forecasts have no systematic pattern.

Considering the conditional coverage test statistic, we see that, for all series, the $p$-values for at least five out of the six specified probabilities for $L=500$ are superior to those for the usual n-GARCH model, while for the n-EGARCH model the $p$-values for at least four out of the six cases for both window lengths are supe- rior to those for the usual n-EGARCH model. Summarizing the results for the conditional coverage, the bias-correction method can improve the n-GARCH and n-EGARCH VaR forecasts so much that the VaR predictions acquired are different from the proposed probability.

Comparing the unconditional coverage results for the bias-correction method based on the n-EGARCH model with those based on the n-GARCH model, we 
Table 4. Unconditional coverage, independence, conditional coverage-CAC.

\begin{tabular}{|c|c|c|c|c|c|c|c|c|c|}
\hline \multirow{2}{*}{$\begin{array}{l}\text { VaR } \\
\text { models }\end{array}$} & \multirow{2}{*}{$\hat{f}$} & \multicolumn{3}{|c|}{$p$-values } & \multirow{2}{*}{$\hat{f}$} & \multicolumn{3}{|c|}{$p$-values } & \multirow[b]{2}{*}{$* / * * * * *$} \\
\hline & & $P_{c c}$ & $P_{\text {ind }}$ & $P_{u n}$ & & $P_{c c}$ & $P_{\text {ind }}$ & $P_{u n}$ & \\
\hline & \multicolumn{4}{|c|}{ VaR 5\% } & \multicolumn{4}{|c|}{ VaR 95\% } & \\
\hline$\hat{f}_{N}$ & 0.0760 & $<0.0001$ & 0.5474 & $<0.0001$ & 0.0400 & 0.0335 & 0.1306 & 0.0338 & $4 / 4 / 3$ \\
\hline$\hat{f}_{N-500}^{*}$ & 0.0575 & 0.2389 & 0.4389 & 0.1324 & 0.0520 & 0.0469 & 0.0147 & 0.6834 & $1 / 0 / 0$ \\
\hline$\hat{f}_{E G A R C H}$ & 0.0715 & 0.0001 & 0.4849 & $<0.0001$ & 0.0450 & 0.0951 & 0.0572 & 0.2970 & $3 / 3 / 3$ \\
\hline$\hat{f}_{E G A R C H-500}^{*}$ & 0.0520 & 0.4741 & 0.2495 & 0.6834 & 0.0485 & 0.3093 & 0.1335 & 0.7571 & 0/0/0 \\
\hline \multirow[t]{2}{*}{$\hat{f}_{t-E G A R C H}$} & 0.0750 & $<0.0001$ & 0.3454 & $<0.0001$ & 0.0455 & 0.0981 & 0.0523 & 0.3487 & $3 / 3 / 3$ \\
\hline & \multicolumn{4}{|c|}{ VaR 1\% } & \multicolumn{4}{|c|}{ VaR 99\% } & \\
\hline$\hat{f}_{N}$ & 0.0200 & 0.0001 & 0.1957 & $<0.0001$ & 0.0095 & 0.8045 & 0.5356 & 0.8207 & \\
\hline$\hat{f}_{N-500}^{*}$ & 0.0095 & 0.8045 & 0.5356 & 0.8207 & 0.0145 & 0.1067 & 0.3474 & 0.0581 & \\
\hline$\hat{f}_{\text {EGARCH }}$ & 0.0215 & $<0.0001$ & 0.1643 & $<0.0001$ & 0.0115 & 0.4289 & 0.2617 & 0.5102 & \\
\hline$\hat{f}_{E G A R C H-500}^{*}$ & 0.0135 & 0.2235 & 0.3813 & 0.1353 & 0.0120 & 0.3883 & 0.2873 & 0.3835 & \\
\hline \multirow[t]{2}{*}{$\hat{f}_{t-E G A R C H}$} & 0.0185 & 0.0014 & 0.2313 & 0.0006 & 0.0080 & 0.1875 & 0.1153 & 0.3517 & \\
\hline & \multicolumn{4}{|c|}{ VaR 0.5\% } & \multicolumn{4}{|c|}{ VaR 99.5\% } & \\
\hline$\hat{f}_{N}$ & 0.0100 & 0.0166 & 0.5148 & 0.0053 & 0.0060 & 0.7654 & 0.6920 & 0.5388 & \\
\hline$\hat{f}_{N-500}^{*}$ & 0.0055 & 0.8913 & 0.7156 & 0.7551 & 0.0060 & 0.7654 & 0.6920 & 0.5388 & \\
\hline$\hat{f}_{E G A R C H}$ & 0.0145 & $<0.0001$ & 0.3474 & $<0.0001$ & 0.0055 & 0.8913 & 0.7156 & 0.7551 & \\
\hline$\hat{f}_{E G A R C H-500}^{*}$ & 0.0065 & 0.6037 & 0.6686 & 0.3634 & 0.0050 & 0.9462 & 0.7395 & 1.0000 & \\
\hline$\hat{f}_{t-E G A R C H}$ & 0.0110 & 0.0036 & 0.4744 & 0.0010 & 0.0050 & 0.9462 & 0.7395 & 1.0000 & \\
\hline
\end{tabular}

Note: See the legend of Table 2 for explanations.

conclude that the n-EGARCH model outperforms the n-GARCH model as for all but the NIKKEI index: the $p$-values for at least four out of the six specified probabilities are superior to those for the n-GARCH model with $L=500$. Similar results hold for the conditional coverage test statistic for all series. Additionally, the bias-correction method based on the n-EGARCH model is the only model never rejected by any of the three performance tests of Christoffersen (1998) for all the specified probabilities (even at $99.5 \%$ and $0.5 \%$ tails) and considered indices.

Consequently, the bias-correction method based on the n-EGARCH model leads to an acceptable performance for out-of-sample VaR prediction. In other words, it seems that allowing for an asymmetric response of the conditional variance to positive and negative shocks generally yields improvement in the VaR performance of the bias-correction method. Therefore, 
Table 5. Average score differences and tests of equal predictive accuracy for the censored likelihood (csl).

\begin{tabular}{|c|c|c|c|c|c|c|}
\hline & FTSE & NIKKEI & CAC & FTSE & NIKKEI & CAC \\
\hline & \multicolumn{3}{|c|}{ VaR 5\% } & \multicolumn{3}{|c|}{ VaR 95\% } \\
\hline$d_{N, 500}$ & -0.0507 & -0.0401 & -0.0720 & -0.1252 & -0.2692 & -0.1791 \\
\hline Test stat. & -5.8568 & -4.4134 & -0.0001 & -0.0023 & -0.0004 & -0.0018 \\
\hline$d_{E G A R C H, 500}$ & -0.0569 & -0.0181 & -0.0793 & -0.0179 & -0.1373 & -0.0395 \\
\hline Test stat. & -6.4187 & -4.6009 & -0.0001 & -4.2077 & -0.0002 & -0.0002 \\
\hline$d_{N, E G A R C H-500}$ & -0.0007 & 0.0204 & -0.0059 & -0.0539 & -0.0976 & -0.1273 \\
\hline Test stat. & -8.2003 & 2.6194 & -8.8945 & -6.0130 & -5.2386 & -8.6942 \\
\hline$d_{E G A R C H_{-} t, 500}$ & -0.0259 & 0.0283 & -0.0540 & 0.0519 & -0.1897 & -0.0572 \\
\hline \multirow[t]{2}{*}{ Test stat. } & -2.3995 & 1.8529 & -6.6502 & 8.7729 & -0.0001 & -2.6532 \\
\hline & \multicolumn{3}{|c|}{ VaR 1\% } & \multicolumn{3}{|c|}{ VaR 99\% } \\
\hline$d_{N, 500}$ & -0.0478 & -0.0268 & -0.0521 & -0.4432 & -0.6463 & -0.3534 \\
\hline Test stat. & -5.7177 & -5.3513 & -0.0001 & -0.0010 & -0.0005 & -0.0004 \\
\hline$d_{E G A R C H, 500}$ & -0.0572 & -0.0057 & -0.0392 & -0.2748 & -0.5455 & -0.1229 \\
\hline Test stat. & -3.9568 & -3.9915 & -0.0001 & -0.0007 & -0.0002 & -0.0002 \\
\hline$d_{N, E G A R C H-500}$ & -0.0100 & 0.0031 & 0.0270 & -0.0688 & -0.2125 & -0.2187 \\
\hline Test stat. & -1.3473 & 1.5694 & 7.7423 & -2.0162 & -2.6433 & -4.2149 \\
\hline$d_{E G A R C H \_t, 500}$ & -0.0272 & -0.0002 & -0.0046 & 0.4595 & -0.1076 & 0.3828 \\
\hline \multirow[t]{2}{*}{ Test stat. } & -5.9952 & -3.5245 & -1.2083 & 2.7004 & -2.0393 & 5.2561 \\
\hline & \multicolumn{3}{|c|}{ VaR $0.5 \%$} & \multicolumn{3}{|c|}{ VaR 99.5\% } \\
\hline$d_{N, 500}$ & -0.0358 & -0.0386 & -0.0269 & -0.5041 & -0.7674 & -0.1196 \\
\hline Test stat. & -0.0002 & -7.3189 & -0.0002 & -0.0005 & -0.0004 & -4.7315 \\
\hline$d_{E G A R C H, 500}$ & -0.0446 & -0.0066 & -0.0457 & -0.3150 & -0.5415 & 0.4784 \\
\hline Test stat. & -6.0635 & -4.0795 & -0.0001 & -0.0004 & -0.0003 & 0.0001 \\
\hline$d_{N, E G A R C H-500}$ & -0.0244 & 0.0019 & 0.0079 & -0.0574 & -0.1053 & 0.0808 \\
\hline Test stat. & -3.4634 & 1.6062 & 5.2479 & -1.3984 & -2.5569 & 8.9946 \\
\hline$d_{E G A R C H \_t, 500}$ & -0.0249 & 0.0062 & -0.0116 & 0.7539 & 0.1648 & 1.2717 \\
\hline Test stat. & -8.1588 & 1.0638 & -2.7084 & 2.9685 & 1.3557 & 5.5332 \\
\hline
\end{tabular}

Note: $d_{N, 500}\left(d_{E G A R C H, 500}\right)$ : average score difference based on $\mathrm{n}-\mathrm{AR}(1)-\mathrm{GARCH}(1,1)(\mathrm{n}-\mathrm{AR}(1)$-EGARCH$(1,1))$ model relative to calibrated n-AR(1)-GARCH(1,1) (n-AR(1)-EGARCH(1,1)) model with $L=500 ; d_{N, E G A R C H-500}$ : average score difference for calibrated $\mathrm{n}$ $\operatorname{AR}(1)-G A R C H(1,1)$ relative to calibrated $n-A R(1)-E G A R C H(1,1)$ model with $L=500 ; d_{E G A R C H \_t, 500}$ : average score difference for t$\operatorname{AR}(1)-E G A R C H(1,1)$ model relative to calibrated $n-A R(1)-E G A R C H(1,1)$ model with $L=500$. Additionally, the corresponding test statistics are shown. The results for both long and short positions are reported in the left and right panel of the table, respectively. 

of violations. Additionally, future work could extend the methodology to other types of GARCH models such as the power (G)ARCH models of Brooks, Faff, McKenzie and Mitchell (2000) or several GARCH models considered by Loudon, Watt and Yadav (2000).

\section{References}

Alexander, C. (2001). Market models: A guide to financial data analysis. Chichester, UK: Wiley.

Bams, D., Lehnert, T., \& Wolff, C.C.P. (2005). An evaluation framework for alternative VaR-models. Journal of International Money and Finance, 24(6), 944-958.Bao, Y., Lee, T.H., \& Saltoğlu, B. (2007). Comparing density forecast models. Journal of Forecasting, 26(3), 203-225.

Barone-Adesi, G., Giannopoulos, K., \& Vosper, L. (1999). VaR without correlations for portfolios of derivative securities. Journal of Futures Markets, 19(5), 583-602.

Barone-Adesi, G., Giannopoulos, K., \& Vosper, L. (2002). Backtesting derivative portfolios with filtered historical simulation (fhs). European Financial Management, 8(1), 31-58.

Basle Committee on Banking Supervision. (1995). An internal model-based approach to market risk capital requirements. Retrieved from https://www.bis. org/publ/bcbsc224.pdf

Basle Committee on Banking Supervision. (1996). Overview of the amendment to the capital accord to incorporate market risks. Retrieved from http:// www.bis.org/publ/bcbs23.pdf

Black, F. (1976). Studies in Stock Price Volatility Changes. In Proceedings of the 1976 Business Meeting of the Business and Economic Statistics Section (pp. 177-181). Washington, DC: American Statistical Association.

Bollerslev, T. (1986). Generalized autoregressive conditional heteroskedasticity. Journal of Econometrics, 31, 307-327.

Bollerslev, T., \& Mikkelsen, H. O. (1996). Modeling and pricing long memory in stock market volatility. Journal of Econometrics, 73, 151-184.

Brooks, R.D., Faff, R.W., McKenzie, M.D. \& Mitchell, H. (2000). A multi-country study of power ARCH models and national stock market returns. Journal of International Money and Finance, 19(3), 377397.
Christoffersen, P.F. (1998). Evaluating intervals forecasts. International Economic Review 39(4), 841862.

Christoffersen, P., \& Gonçalves, S. (2005). Estimation risk in financial risk management. Journal of Risk, 7, 1-28.

Crouhy, M., Galai, D., \& Mark, R. (2001). Risk management. New York, NY: McGraw-Hill.

Diks, C., Panchenko, V., \& van Dijk, D. (2011). Likelihood-based scoring rules for comparing density forecasts in tails. Journal of Econometrics, 163(2), 215-230.

Ding, Z., Granger, C.W.J., \& Engle, R.F. (1993). A long memory property of stock market returns and a new model. Journal of Empirical Finance, 1(1), 83-106.

Dowd, K. (2005). Measuring market risk (2 ${ }^{\text {nd }}$ ed.). Chichester, UK: John Wiley \& sons, Ltd.

Engle, R.F. (1982). Autoregressive conditional heteroscedasticity with estimates of the variance of United Kingdom inflation. Econometrica, 50(4), 987-1008.

Engle, R.F., \& Ng, V. (1993). Measuring and testing the impact of news on volatility. The Journal of Finance, 48(5), 1749-1778.

Fama, E.F. (1965). The behavior of stock market price. Journal of Business, 38(1), 34-105.

Giot, P., \& Laurent, S. (2003). Value-at-risk for long and short trading positions. Journal of Applied Econometrics, 18(6), 641-663.

Glosten, L.R., Jagannathan, R., \& Runkle, D.E. (1993). On the relation between the expected value and the volatility of the nominal excess return on stocks. The Journal of Finance, 5(48), 1779-1801.

Hartz, C., Mittnik, S., \& Paolella, M. (2006). Accurate value-at-risk forecasting based on the normalGARCH model. Computational Statistics \& Data Analysis, 51(4), 2295-2312.

Kupiec, P.H. (1995). Techniques for verifying the accuracy of risk measurement models. The Journal of Derivatives, 3(2), 73-84.

Loudon, G.F., Watt, W.H., \& Yadav, P.K. (2000). An empirical analysis of alternative parametric ARCH model. Journal of Applied Econometrics, 15(2), 117-136.

Nelson, D.B. (1991). Conditional heteroskedasticity in asset returns: A new approach. Econometrica, 59(2), 347-370. 
Nieto, M.R., \& Ruiz, E. (2010). Bootstrap prediction intervals for VaR and ES in the context of GARCH models (Working Paper No. 10-28). Departamento de Estadística, Universidad Carlos III de Madrid. Retrieved from http://earchivo.uc3m. es/bitstream/handle/10016/8524/ws 102814. pdf? sequence $=1$

Pascual, L., Romo, J., \& Ruiz, E. (2006). Bootstrap prediction for returns and volatilities in GARCH models. Computational Statistics \& Data Analysis, 50(9), 2293-2312.

Reeves, J. (2005). Bootstrap prediction intervals for ARCH models. International Journal of Forecasting, 21(2), 237-248.

Trucíos, C., \& Hotta, L. (2016). Bootstrap prediction in univariate volatility models with leverage effect. Mathematics and Computers in Simulation, 120, 91-103.

Tsay, R.S. (2005). Analysis of financial time series. New Jersey, NJ: A John Wiley \& Sons Inc.

\section{Endnotes}

1 For the sake of brevity, only the results for Student's $t$ distribution are presented in this paper. The results for the GED, which are very similar to those for the Student's $t$ distribution, are available from the authors upon request.

2 For a more technical definition of the method, please refer to Diks et al. (2011). 
